\title{
The brain is processing information, not data. Does anybody care?
}

\author{
Emanuel Diamant 1 \\ ${ }^{1}$ VIDIA-mant/ POB 933, Kiriat Ono 5510801, Israel \\ E-Mails: eman1.245@gmail.com;
}

\section{Extended Abstract}

Despite what is claimed in the title, it is generally agreed that the brain is processing data, not information. The source of this mismatch is twofold: First, the reality of the early 1950s, when digital computers have become a part of our life and the concept of the brain as a computer has become popular and ubiquitous. Computer is a data-processing machine. Consequently, the brain was announced as a data-processing device.

On the other hand, exactly at the same time, as a part of his Mathematical Theory of Communication, Claude Shannon has coined the notion of "information", [1]. Concerned with delivering a message from a sender to a receiver (through an unreliable and noisy communication channel), Shannon defines information as an equivalent to the surprise value of the message. Defining information in terms of a signal transmission (that is, data transition), while totally neglecting the meaning of the message (that is, information associated with the message delivery) was from the beginning recognized as a deficit of the Shannon's theory. Nevertheless, the success of Shannon's information as a tool for the technical telecommunication problems solution, made Shannon's information concept accepted and shared by almost all other scientific communities. However, the popularity of its use has resulted in a widespread blur and confusion between the terms "data" and "information", and the most of the scientific community does not differentiate or distinguish between the two today. As a rule, the terms are used interchangeably and improperly.

In attempt to avoid this unpleasant situation, I propose a new definition of information more suitable for the purposes of brain and other biotic systems research. The definition is an extended version of the Kolmogorov's mid-60s definition [2] that sounds like this:

\section{"Information is a linguistic description of structures observable in a given data set".}

A digital image can serve us for a definition "examination". An image is a two-dimensional set of data elements called pixels. In an image, pixels are distributed not randomly, but due to the similarity in their physical properties, and are naturally grouped into some clusters or clumps. I propose to call these clusters primary or physical data structures.

In the eyes of an external observer, the primary data structures are further arranged into more larger and complex assemblies, which I propose to call secondary data structures. These secondary structures 
reflect human observer's view on the primary data structures composition, and therefore they could be called meaningful or semantic data structures. While formation of primary data structures is guided by objective (natural, physical) properties of the data, ensuing formation of secondary structures is a subjective process guided by human habits and customs.

As it was said, Description of structures observable in a data set should be called "Information". In this regard, two types of information must be distinguished - Physical Information and Semantic Information. They are both language-based descriptions; however, physical information can be described with a variety of languages (recall that mathematics is also a language), while semantic information can be described only by means of the natural human language. (More details on the subject are in [3]).

One can hardly overestimate the importance of physical and semantic information segregation. For the first time, data-based information and its semantic (language-based) interpretation are detached and now can be treated correctly and effectively.

For the first time, information is represented as a linguistic description, as a string of words, a piece of text. It does not matter that in biotic applications these texts are written in the four-letter nucleotide alphabet. The important thing is that now information is materialized, and as such can be stored, retrieved, changed, transmitted and (generally speaking) processed as any other material object.

In this way, thoughts and memories are now become materialized too, and the thinking process (as all other brain activities) must be seen now as a text processing activity.

Contemporary computers are data-processing machines and thus are not suitable for such text (semantic information) processing. The brain is doing all this effortless and efficiently, that is, effortless and efficiently it is processing information, and not data (as it is correctly specified in the title). How does it do this? - that is a good question. But with the right understanding about what information is, it will be easier for us to reach the right answer.

\section{References}

1. Shannon, C., Weaver, W.; The Mathematical Theory of Communication, University of Illinois Press, 1949. http://raley.english.ucsb.edu/wp-content/Engl800/Shannon-Weaver.pdf

2. Kolmogorov, A; Three approaches to the quantitative definition of information, Problems of Information and Transmission, Vol. 1, No. 1, pp. 1-7, 1965.

http://alexander.shen.free.fr/library/Kolmogorov65_Three-Approaches-to-Information.pdf

3. Diamant, E.; Brain, Vision, Robotics and Artificial Intelligence. http://www.vidia-mant.info 